Pacific Journal of Mathematics

THE RANGE OF CONVOLUTION OPERATORS 


\title{
THE RANGE OF CONVOLUTION OPERATORS
}

\author{
ROBERT A. BEKES
}

\begin{abstract}
Subject to certain restrictions, convolving on the right by a fixed function defines a bounded linear operator between spaces of measures or functions over a locally compact group. For non-compact groups we show that when the range and domain are different, such operators rarely have closed range. Applications of these results are made to representation theory for locally compact groups. We also prove a correspondence theorem for strictly cyclic vectors for Banach algebras and those for certain closed left ideals.
\end{abstract}

Preliminaries. Throughout this paper $G$ denotes a locally compact, non-compact group with left Haar measure $d x$. Let $M(G)$ denote the space of finite Borel measures with total variation norm $\|\cdot\| ; L_{p}(G)$, $1 \leq p<\infty$, the equivalence classes of $p$-integrable functions with norm $\|\cdot\|_{p} ; L_{\infty}(G)$ the essentially bounded measurable functions on $G$ with the essential supremum norm $\|\cdot\|_{\infty} ; C(G)$ the bounded continuous functions on $G$ with the uniform norm also denoted by $\|\cdot\|_{\infty} ; C_{0}(G)$ those $f$ in $C(G)$ that vanish at infinity; $C_{00}(G)$ those $f$ in $C_{0}(G)$ with compact support. See Hewitt and Ross for formal definitions of these objects.

For a function $f$ on $G$ and a fixed $x$ in $G$ the left translation of $f$ by $x$, written $x f$, is defined by $x f(y)=f(x y)$ for all $y$ in $G$. Note that if the support of $f$ is $K$, then the support of $x f$ is $x^{-1} K$. Let $\tilde{f}$ denote the function $\tilde{f}(x)=f\left(x^{-1}\right)$. Continuity of the group operations implies that $f$ has compact support if and only if $\tilde{f}$ does. If $A$ is a set of functions on $G$, then $\tilde{A}=\{\tilde{f}: f \in A\}$. The complex conjugate of $f$, written $\bar{f}$, is defined by $\bar{f}(x)=\overline{f(x)}$.

Convolution between a measure $\mu$ and a function $f$, when defined, is given by the following formula:

$$
\mu * f(x)=\int f\left(y^{-1} x\right) d \mu(y) .
$$

And when defined between two functions of $f$ and $g$ is given by

$$
g * f(x)=\int f\left(y^{-1} x\right) g(y) d y .
$$

We will need to refer to the following convolution formulas and their associated norm inequalities. See Hewitt and Ross [6, 20.19]. 
(1) $\quad L_{1}(G) * L_{p}(G) \subset L_{p}(G), \quad p \geq 1$ and $\|g * f\|_{p} \leq\|g\|_{1}\|f\|_{p}$.

(2) $M(G) * L_{p}(G) \subset L_{p}(G), \quad p \geq 1$ and $\|\mu * f\|_{p} \leq\|\mu\|\|f\|_{p^{\prime}}$.

(3) $L_{p}(G) *\left[L_{p}(G)\right]^{\sim} \subset C_{0}(G)$, for $1<p<\infty, 1 / p+1 / p^{\prime}=1$ and $\|g * \tilde{f}\|_{\infty} \leq\|g\|_{p}\|f\|_{p^{\prime}}$.

(4) $\quad L_{1}(G) * L_{\infty}(G) \subset C(G)$ and $\|g * f\|_{\infty} \leq\|g\|_{1}\|f\|_{\infty}$.

(6) $L_{p}(G) *\left\{L_{q}(G) \cap\left[L_{q}(G)\right]^{\sim}\right\} \subset L_{r}(G)$

where $p, q>1,1 / p+1 / q-1 / r=1$ and $\|g * f\|_{r} \leq\|g\|_{p}\|f\|_{q}$.

One more convolution formula is needed. It turns out to be an easy consequence of (3) and (4).

$$
L_{1}(G) * C_{0}(G) \subset C(G) \text { and }\|g * f\|_{\infty} \leq\|g\|_{1}\|f\|_{\infty} .
$$

Proof. Let $f \in L_{1}(G)$ and $g \in C_{0}(G)$. Choose $g_{n}, f_{n} \in C_{00}(G)$ such that $\left\|g-g_{n}\right\|_{\infty} \rightarrow 0$ and $\left\|f-f_{n}\right\|_{1} \rightarrow 0$. Now since for any $p, C_{00}(G) \subset$ $L_{p}(G)$ and $\tilde{f}_{n} \in C_{00}(G)$, we have by (3) that $g_{n} * f_{n} \in C_{00}(G)$ for all $n$. By (4), $g * f \in C(G)$ and so

$$
\begin{aligned}
\left\|g * f-g_{n} * f_{n}\right\|_{\infty} & \leq\left\|g * f-g_{n} * f\right\|_{\infty}+\left\|g_{n} * f-g_{n} * f_{n}\right\|_{\infty} \\
& \leq\left\|g-g_{n}\right\|_{1}\|f\|_{\infty}+\left\|g_{n}\right\|_{1}\left\|f-f_{n}\right\|_{\infty} \rightarrow 0 .
\end{aligned}
$$

Therefore $g * f \in C_{0}(G)$. The norm inequality follows from (4) since $C_{0}(G) \subset L_{\infty}(G)$.

Next we collect some facts about linear maps and adjoints. Let $M$ be a normed linear space. We will write $M^{*}$ for the dual space of $M$. If $\varphi \in M^{*}$, the norm of $\varphi$ will be written as $\|\varphi\|_{M^{*}}$. For $\varphi \in M^{*}$ and $m \in M$ it will sometimes be convenient to write $\varphi(m)$ as a bilinear form, $\varphi(m)=$ $\langle m, \varphi\rangle$. If $X$ and $Y$ are Banach spaces and $T: X \rightarrow Y$ is a continuous linear map the adjoint of $T$, denoted by $T^{*}$, is defined on $Y^{*}$ by $\left\langle x, T^{*} y^{*}\right\rangle=\left\langle T x, y^{*}\right\rangle$ for all $x \in X$. It follows from the continuity of $T$ that $T^{*}: Y^{*} \rightarrow X^{*}$. A fact that will be used repeatedly is that if $T: X \rightarrow Y$ is continuous, linear, one-to-one and if the range of $T$ is closed, then $T^{*}$ maps $Y^{*}$ onto $X^{*}$. See Hewitt and Ross [7, E.8].

1. Operators from $L_{1}(G)$ into $L_{p}(G)$ and $C(G)$. Let $f$ be fixed function and $T_{f}$ the operator obtained by convolving on the right by $f$. If $f$ belongs to $L_{p}(G), p \geq 1$, then (1) implies that $T_{f}$ is a bounded operator 
from $L_{1}(G)$ into $L_{p}(G)$. If $f$ belongs to $C_{0}(G)$, it follows from (7) that $T_{f}$ is a bounded operator from $L_{1}(G)$ into $C_{0}(G)$.

In this section we will be concerned with the question of when these operators have closed range. The two cases, $f$ in $L_{p}(G)$ and $f$ in $C_{0}(G)$, are considered together because the main computations in both cases are similar and can conveniently be combined as the following result.

LEMMA 1.1. Let $f \in L_{p}(G), p>1$, or $C_{0}(G), f \neq 0$, and let $M=$ $L_{1}(G)_{\tilde{f}}^{*} f$. Then there exists $\left\{\varphi_{n}\right\}_{n=1}^{\infty} \subset M^{*}$ such that $\left\|\varphi_{n}\right\|_{M^{*}} \rightarrow \infty$ and $\left\|\varphi_{n} * \tilde{\tilde{f}}\right\|_{\infty}$ is bounded.

Proof. Note that implicit in the conclusion of the lemma is that $\varphi_{n}$ can be convolved with $\tilde{\tilde{f}}$. In general there are functionals in $m^{*}$ for which this convolution is not defined in the usual way.

We have two cases to consider: case (i). $f \in L_{p}(G), 1<p<\infty$; and case (ii), $f \in C_{0}(G)$.

Since $C_{0}(G) \subset L_{\infty}(G)$, the uniform norm on $C_{0}(G)$ will sometimes be written as $\|\cdot\|_{p}$ where $p=\infty$. The conjugate exponents are denoted by $p^{\prime}$ where $p^{\prime}=p /(1-p)$ if $1<p<\infty$ and $p^{\prime}=1$ if $p=\infty$.

For the remainder of this lemma fix an integer $n \geq 1$ and $k \in M$, where $\|k\|_{p}=1$.

In case (i) an application of the Hahn-Banach theorem gives us $h \in L_{q}(G)$ such that $\int k(x) \bar{h}(x) d x=1$ and $\|h\|_{p^{\prime}}=1$. In case (ii), since $M(G)=C_{0}(G)^{*}$ and $L_{1}(G)$ is weak* dense in $M(G)$, see Dunford and Schwartz [3], there exists $h \in L_{1}(G)$ such that $\int k(x) \overline{h(x)} d x=1$.

Choose $k_{0} \in C_{00}(G)$ such that $\left\|k-k_{0}\right\|_{p}<1 / n$. Let $A_{0}=$ support of $k_{0}$. In case (i) $k \in L_{p}(G)$ and $h \in L_{p^{\prime}}(G)$ so (3) implies that $\bar{h} * \tilde{k} \in C_{0}(G)$. In case (ii) $k \in C_{0}(G)$ and $h \in L_{1}(G)$ so by (7) we also have that $\bar{h} * \tilde{k} \in C_{0}(G)$. Therefore in either case we can find a compact set $A_{1}$ such that $|\bar{h} * \tilde{k}(x)|<1 / 2 n(n-1)$ for all $x \notin A_{1}$. The same argument as above, this time applied to $h$ and $\bar{f}$ implies that in cases (i) and (ii), $h * \tilde{\tilde{f}}$ belongs to $C_{0}(G)$. So for any fixed $p, 1<p \leq \infty$, there exists $g \in C_{00}(G)$ such that $\|h * \tilde{\tilde{f}}-g\|_{\infty}<\|f\|_{p} / n$. Let $A_{2}=$ support of $g$ and let $B=A_{0}$ $\cup A_{1} \cup A_{2} \cup\{e\}$, where $e$ is the identity element of $G$. Then $B$ is compact. Since $e \in B, B \subset B B^{-1}$ and so $A_{i} \subset B B^{-1}$ for $i=0,1,2$. The continuity of the group operations implies that $B B^{-1}$ is compact and hence so is any translation of $B B^{-1}$.

Next we choose elements $x_{1}, \ldots, x_{n}$ in $G$ by induction as follows. Let $x_{1}$ be any element of $G$. Since $G$ is not compact the set $G \backslash \cup_{j=1}^{l-1} B B^{-1} x_{j}$ is not empty for $i \geq 2$. So let $x_{i} \in G \backslash \cup_{j=1}^{i-1} B B^{-1} x_{j}$. We derive some 
properties of $x_{1}, \ldots, x_{n}$. Since $x_{j} \notin B B^{-1} x_{i}$ for $i<j$ we have that $B^{-1} x_{j} \cap$ $B^{-1} x_{i}=\varnothing$ for $i<j$. And so $x_{j}^{-1} B \cap x_{i}^{-1} B=\varnothing$ for all $i \neq j$. Therefore $x_{j}^{-1} A_{m} \cap x_{1}^{1} A_{m}=\varnothing$ for $i \neq j$ and $m=0,1,2$. This implies, in particular, that the supports of the functions $x_{i} k_{0}$ are disjoint.

In case (i), where $1<p<\infty$, we get

$$
\left\|\sum_{i=1}^{n} x_{i} k_{0}\right\|_{p}^{p}=\sum_{i=1}^{n}\left\|x_{i} k_{0}\right\|_{p}^{p} \leq 2^{p} n .
$$

In case (ii), where $p=\infty$, we get

$$
\left\|\sum_{i=1}^{n} x_{i} k_{0}\right\|_{\infty}=\left\|k_{0}\right\|_{\infty} \leq 2
$$

We also have that

$$
\begin{aligned}
\left\langle x_{j} k, x_{i} h\right\rangle & =\int \overline{x_{i} h}(x) x_{j} k(x) d x \\
& =\int \bar{h}\left(x_{i} x\right) k\left(x_{j} x\right) d x=\int \bar{h}(x) k\left(x_{j} x_{i}^{-1} x\right) d x \\
& =\int \bar{h}(x) \tilde{k}\left(x^{-1} x_{i} x_{j}^{-1}\right) d x=\bar{h} * \tilde{k}\left(x_{i} x_{j}^{-1}\right) .
\end{aligned}
$$

The $x_{i}$ were chosen so that $x_{i} x_{j}^{-1} \notin B B^{-1}$ for $i \neq j$ and, since $A_{1} \subset B B^{-1}$, we get for $i \neq j$ that

$$
\left|\int x_{i} \bar{h}(x) x_{j} k(x) d x\right|=\left|\bar{h} * \tilde{k}\left(x_{i} x_{j}^{-1}\right)\right|<1 / 2 n(n-1) .
$$

Let $\varphi_{n}=\sum_{i=1}^{n} x_{i} h$. Then $\varphi_{n} \in M^{*}$ and

$$
\begin{aligned}
\left|\left\langle\sum_{j=1}^{n} x_{j} k, \varphi_{n}\right\rangle\right| & =\left|\int \bar{\varphi}_{n}(x) \sum_{j=1}^{n} x_{j} k(x) d x\right| \\
& =\left|\int\left[\sum_{j=1}^{n} x_{i} \bar{h}(x)\right]\left[\sum_{j=1}^{n} x_{j} k(x)\right] d x\right| \\
& =\left|\sum_{i=1}^{n} x_{i} \bar{h}(x) x_{i} k(x) d x+\sum_{i \neq j} x_{i} \bar{h}(x) x_{j} k(x) d x\right| \\
& =\left|n \int \bar{h}(x) k(x) d x+\sum_{i \neq j} \bar{h} * k\left(x_{i} x_{j}^{-1}\right)\right| \\
& \geq n-n(n-1) / 2 n(n-1) \geq n / 2 .
\end{aligned}
$$


We note here that $M$ is closed under left translation. This fact follows from the following relation:

$$
x(g * f)=(x g) * f \text { for all } x \in G, g \in L_{1}(G) .
$$

Therefore $\sum_{j=1}^{n} x_{j} k \in M$. Now

$$
\begin{aligned}
\left\|\sum_{j=1}^{n} x_{j} k\right\|_{p} & \leq\left\|\sum_{j=1}^{n} x_{j} k-\sum_{j=1}^{n} x_{j} k_{0}\right\|_{p}+\left\|\sum_{j=1}^{n} x_{j} k_{0}\right\|_{p} \\
& \leq n\left(\frac{1}{n}\right)+\left\|\sum_{j=1}^{n} x_{j} k_{0}\right\|_{p} .
\end{aligned}
$$

In case (i), where $1<p<\infty$, we get from (9) that

$$
\left\|\sum_{j=1}^{n} x_{j} k\right\|_{p} \leq 1+2 n^{1 / p} \leq 3 n^{1 / p}
$$

Therefore a lower bound for $\left\|\varphi_{n}\right\|_{M^{*}}$ can be computed as

$$
\left\|\varphi_{n}\right\|_{M^{*}} \geq \mid\left\langle\frac{1}{3 n^{1 / p}} \sum_{j=1}^{n} x_{j} k, \varphi_{n}\right\rangle \geq\left(\frac{1}{3 n^{1 / p}}\right) \frac{n}{2}=\frac{n^{1 / q}}{6} .
$$

In case (ii), where $p=\infty$, we get from (10) that $\left\|\sum_{j=1}^{n} x_{j} k\right\|_{\infty} \leq 3$ and so a lower bound for $\left\|\varphi_{n}\right\|_{M^{*}}$ is

$$
\left\|\varphi_{n}\right\|_{M^{*}} \geq \mid\left\langle\frac{1}{3} \sum_{j=1}^{n} x_{j} k, \varphi_{n}\right\rangle \geq\left(\frac{1}{3}\right) \frac{n}{2}=\frac{n}{6} .
$$

Next we compute the norm of $\varphi_{n} * \tilde{\tilde{f}}$. Note that in both cases $\underline{\underline{f}}_{n} * \tilde{\tilde{f}}$ belongs to $C_{0}(G)$ since it is a linear combination of translates of $h * \tilde{\tilde{f}}$.

By (8) we have $x_{j}^{-1} A_{2} \cap x_{i}^{-1} A_{2}=\varnothing$ for $i \neq j$ and so the supports of the functions $x_{i} g$ are disjoint. Therefore $\left\|\sum_{i=1}^{n} x_{i} g\right\|_{\infty}=\|g\|_{\infty}$. We also have that

$$
\|g\|_{\infty} \leq\|g-h * \tilde{\tilde{f}}\|_{\infty}+\|h * \tilde{\tilde{f}}\|_{\infty} .
$$

An application of (3) when $1<p<\infty$ and an application of (7) when $p=\infty$ yields $\|h * \tilde{\tilde{f}}\|_{\infty} \leq\|h\|_{p^{\prime}}\|f\|_{p}$. So from above,

$$
\|g\|_{\infty} \leq\|f\|_{p}+\|h\|_{p^{\prime}}\|f\|_{p} .
$$


In either case,

$$
\begin{aligned}
\left\|\varphi_{n} * \tilde{\tilde{f}}-\sum_{i=1}^{n} x_{i} g\right\|_{\infty} & =\left\|\sum_{i=1}^{n}\left(x_{i} h\right) * \tilde{\tilde{f}}-\sum_{i=1}^{n} x_{i} g\right\|_{\infty} \\
& =\left\|\sum_{i=1}^{n} x_{i}(h * \tilde{\bar{f}})-\sum_{i=1}^{n} x_{i} g\right\|_{\infty} \\
& \leq \sum_{i=1}^{n}\left\|x_{i}(h * \tilde{\tilde{f}})-x_{i} g\right\|_{\infty}<\|f\|_{p} .
\end{aligned}
$$

Putting these inequalities together yields

$$
\begin{aligned}
\left\|\varphi_{n} * \tilde{\tilde{f}}\right\|_{\infty} & \leq\left\|\varphi_{n} * \tilde{\tilde{f}}-\sum_{i=1}^{n} x_{i} g\right\|_{\infty}+\left\|\sum_{i=1}^{n} x_{i} g\right\|_{\infty} \\
& \leq\left[2+\|h\|_{p^{\prime}}\right]\|f\|_{p} .
\end{aligned}
$$

Keeping $k$ and $h$ fixed for all $n$, we see that $\varphi_{n}$ depends only on $n$. So the lower bounds in (11) and (12) hold for each $n$, while the bound in (13) is independent of $n$. This proves the lemma.

Note that when the operator $T_{f}$ (i.e., convolution on the right by $f$ ) is defined on $L_{1}(G)$, its range is the set $L_{1}(G) * f$.

THEOREM 1.1. (i) Let $p>1, f \in L_{p}(G)$ and $f \neq 0$. Then $L_{1}(G) * f$ is not closed in $L_{p}(G)$.

(ii) Let $f \in C_{0}(G), f \neq 0$. Then $L_{1}(G) * f$ is not closed in $C_{0}(G)$.

Proof. (i) The remarks prior to Lemma 1.1 show that $T_{f}$ is a bounded operator from $L_{1}(G)$ into $L_{p}(G)$ with range $L_{1}(G) * f$. Let $M=L_{1}(G) * f$ and suppose $M$ is closed in $L_{p}(G)$. Since $T_{f}$ is continuous, $\mathcal{L}=T_{f}^{-1}\{0\}$ is a closed subspace of $L_{1}(G)$. Consider the map $\Phi: L_{1}(G) / \mathcal{L} \rightarrow L_{p}(G)$ defined by $\Phi(g+\mathcal{L})=g * f$. Then $\Phi$ is well defined and one-to-one. We show that $\Phi$ is bounded. Let $h \in \mathcal{L}$; then

$$
\begin{aligned}
\|\Phi(g+\mathcal{L})\| & =\|g * f\|_{p}=\|g * f-h * f\|_{p} \\
& =\|(g-h) * f\|_{p} \leq\|g-h\|_{1}\|f\|_{p} .
\end{aligned}
$$

So taking the infimum over $h$ in $\mathcal{L}$ we get $\|\Phi(g+\mathcal{L})\| \leq\|g+\mathcal{L}\|\|f\|_{p}$. Since the range of $\Phi$ is $M$, which we assumed to be closed, the open mapping theorem gives us that $\Phi$ is a bicontinuous map from $L_{1}(G)$ onto $M \subset L_{p}(G)$. As we noted in the preliminaries, the adjoint map of $\Phi, \Phi^{*}$, will then map $M^{*}$ onto $\left(L_{1}(G) / \mathcal{L}\right)^{*}$ and, since $\Phi$ is bicontinuous, so is $\Phi^{*}$. Now $M$ is embedded as a closed subspace of $L_{p}(G)$ under the identity 
map $i$. It follows that the adjoint map, $i^{*}$, maps $\left(L_{p}(G)\right)^{*}$ onto $M^{*}$. But $\left(L_{p}(G)\right)^{*}$ is isomorphic to $L_{p^{\prime}}(G), 1 / p+1 / p^{\prime}=1$. So we can identify $M^{*}$ with all those elements of $L_{p^{\prime}}(G)$ which give rise to non-zero functionals on $M$. Furthermore, it follows from Dunford and Schwartz [3, II, 4.18b] that $\left(L_{1}(G) / \mathfrak{L}\right)^{*}$ is isometrically isomorphic with the closed subspace, $\mathcal{L}^{\perp}$, of $L_{\infty}(G)$, where $\mathcal{L}^{\perp}=\left\{k \in L_{\infty}(G):\langle g, k\rangle=0\right.$ for all $\left.g \in \mathcal{L}\right\}$. Therefore $\Phi^{*}$ can be realized as bicontinuous from $M^{*}$ onto $\mathcal{L}^{\perp}$. For an arbitrary $h$ in $L_{q}(G)$ we compute $\Phi^{*}(k)$. Since $\Phi^{*}(h) \in L_{\infty}(G)$ and $L_{\infty}(G)$ is isomorphic to $\left(L_{1}(G)\right)^{*}$, we compute how $\Phi^{*}(h)$ acts as a functional on $L_{1}(G)$. So let $g \in L_{1}(G)$ be arbitrary. Then

$$
\begin{aligned}
\int g(x) \overline{\Phi^{*}(h)}(x) d x & =\langle\Phi(g+\mathfrak{L}), h\rangle=\langle g * f, h\rangle \\
& =\int g * f(x) \bar{h}(x) d x \\
& =\iint g(y) f\left(y^{-1} x\right) \bar{h}(x) d y d x \\
& =\iint g(y) \bar{h}(x) \tilde{f}\left(x^{-1} y\right) d x d y \\
& =\int g(y)\left[\int h(x) \tilde{\tilde{f}}\left(x^{-1} y\right) d x\right] \bar{d} y \\
& =\int g(y)[h * \tilde{\tilde{f}}(y)]^{-} d y .
\end{aligned}
$$

It follows that $\Phi^{*}(h)=h * \tilde{\tilde{f}}$. But by Lemma 1.1 there exists $\left\{\varphi_{n}\right\} \subset M^{*}$ such that $\left\|\varphi_{n}\right\|_{M^{*}} \rightarrow \infty$ and $\left\|\Phi^{*}\left(\varphi_{n}\right)\right\|_{\infty}$ is bounded. This contradicts the bicontinuity of $\Phi^{*}$ and so contradicts the assumption that $M$ is closed in $L_{p}(G)$. This proves part (i) of the theorem.

The proof of part (ii) is the same as that for part (i) except that here $M=L_{\mathrm{l}}(G) * f$ is a subspace of $C_{0}(G)$. We define $\mathcal{L}$ as in part (i) and we see that $\Phi$ maps $L_{1}(G) / \mathcal{L}$ onto $M$ from which it follows that $\Phi^{*}$ maps $M^{*}$ to $\mathfrak{L}^{\perp}$. Now if $M$ is assumed to be closed in $C_{0}(G), \Phi^{*}$ will then be bicontinuous. Now $M^{*}$ can be identified with those measures in $M(G)=$ $\left(C_{0}(G)\right)^{*}$ which don't vanish on $M$ and a computation similar to that in part (i) shows that for $\mu \in M(G), \Phi^{*}(\mu)=\mu * \tilde{\tilde{f}}$. At this point Lemma 1.1 can be used to contradict the bicontinuity of $\Phi^{*}$ and therefore the assumption that $M$ is closed in $C_{0}(G)$ can't hold. This proves part (ii) of the theorem. 
EXAMPLE 1.1. When $p=1$ the theorem is not true in general. Let $G=\mathbf{Z}$, the integers, and let $\boldsymbol{\delta}_{0}$ be point mass at 0 . Then $l_{1}(\mathbf{Z}) * \delta_{0}=l_{1}(\mathbf{Z})$. For an example of a non-discrete group where the theorem fails to hold when $p=1$ is $G=\mathbf{Z} \times T$, the integers cross the circle group and $f$ the characteristic function of $\{0\} \times T$. Then it's easy to see that $L_{1}(\mathbf{Z} \times T) * f$ is closed in $L_{1}(\mathbf{Z} \times T)$ since $f$ is idempotent.

For further discussion of the problem of when $\mu * L_{1}(G)$ is closed see I. Glicksberg's paper [4].

2. An application to representation theory. Let $\pi$ be a continuous unitary representation of $G$ on a Hilbert space $H$. See Hewitt and Ross [6, $\S 21]$. Then $\pi$ can be extended to a continuous representation of $L_{1}(G)$ as bounded operators on $H$ by the following formula: For $f \in L_{1}(G)$, and $\xi, \eta \in H, \pi(f)$ is the operator defined by

$$
\langle\pi(f) \xi, \eta\rangle=\int f(x)\langle\pi(x) \gamma x, \eta\rangle d x
$$

It turns out that $\pi$ is a continuous algebra homorphism of $L_{1}(G)$ into the bounded operators on $H$. We call a vector $\xi \in H$ strictly cyclic for $\pi$ if $\left\{\pi(g) \xi: g \in L_{1}(G)\right\}=H$, and we say that $\pi$ is algebraically irreducible if every non-zero vector in $H$ is strictly cyclic for $\pi$.

When $H=L_{2}(G)$ and $\pi(x) f=x^{-1} f$ for $f \in L_{2}(G)$ then $\pi$ is called the left regular representation of $G$. It follows that $\pi(g) f=g * f$ for $g \in L_{1}(g)$ and $f \in L_{2}(G)$. In Bekes [2] it is shown that no non-zero subrepresentation of the left regular representation of a locally compact, non-compact group can be algebraically irreducible. Note that $f \in L_{2}(G)$ will be strictly cyclic for a subrepresentation of the left regular representation if and only if $L_{1}(G) * f$ is closed in $L_{2}(G)$. Therefore a more general result follows immediately from Theorem 1.1(i) when $p=2$ :

COROllary 2.1. No non-zero subrepresentation of the left regular representation of a locally compact, non-compact group can have a strictly cyclic vector.

A characterization of compact groups in terms of the existence of a strictly cyclic vector is possible.

For compact groups the left regular representation decomposes into a direct sum of irreducible finite-dimensional subrepresentations. And since every non-zero vector in an irreducible finite-dimensional representation is strictly cyclic, a consequence of Corollary 2.1 is the following. 
COROLLARY 2.2. A locally compact group is compact if and only if some non-zero subrepresentation of the left regular representation has a strictly cyclic vector.

Cyclic vectors for representations of groups have been studied by $\mathrm{F}$. Greenleaf and M. Moskowitz in [5].

3. Maps from $M(G)$ into $L_{p}(G)$ and $C_{0}(G)$. We extend Theorem 1.1 to maps from $M(G)$ into $L_{p}(G), 1<p<\infty$ or $C_{0}(G)$. In doing this we need a result on the correspondence between strictly cyclic vectors for Banach algebras and those of closed ideals which have bounded approximate identities. We prove a more general result in that we only assume the ideal is one sided.

To begin we record a lemma due to Bade and Curtis [1, 1.2].

LeMma 3.1. Let $T$ be a bounded linear map from $X$ into $Y$ where $X$ is a Banach space. Suppose there exists $c>0$ and $0 \leq \alpha<1$ such that given $y \in Y,\|y\| \leq 1$, there exists $x \in X,\|x\| \leq c$, such that $\|T x-y\|<\alpha$. Then $T$ is subjective.

Let $\mathfrak{U}$ be a Banach algebra and $X$ a Banach space. Suppose there is a mapping of $\mathfrak{A} \times X$ into $X$, the image of $(a, x)$ denoted by $a x$, such that for $\alpha \in \mathbf{C}, a, b \in \mathfrak{A}, x \in X$ :

(i) $(\alpha a) x=\alpha(a x)=a(\alpha x)$;

(ii) $(a b) x=a(b x)$;

(iii) there exists $K>0$ such that $\|a x\| \leq K\|a\|\|x\|$;

then $X$ is called a left Banach $\mathfrak{A}$ module (see Hewitt and Ross [7, 32.14]).

A net $\left\{h_{\delta}\right\}_{\delta \in \Lambda}$ in a normed algebra $B$ is called a bounded left approximate identity if given any $b \in B,\left\|h_{\delta} b-b\right\| \rightarrow 0$ and $\sup _{\delta}\left\|h_{\delta}\right\|<$ $\infty$.

LEMma 3.2. Let $\mathfrak{Y}$ be a Banach algebra and $X$ a left Banach module. Suppose $\mathcal{G}$ is a closed left ideal of $\mathfrak{A}$ which as a Banach algebra has a bounded left approximate identity. Let $x \in \overline{\bar{X} X}$. Then $\mathfrak{A} x$ is closed if and only if $q x$ is closed and they are equal.

Proof. By the factorization theorem for left Banach modules, Hewitt and Ross [7, 32.22], we have $g X$ closed in $X$ and there exists $a_{0} \in \mathcal{G}$ and $x_{0} \in X$ such that $x=a_{0} x_{0}$. Let $\left\{h_{\delta}\right\}_{\delta \in \Lambda}$ be a bounded left approximate 
identity for $q$. Then

$$
\left\|h_{\delta} x-x\right\|=\left\|h_{\delta} a_{0} x_{0}-a_{0} x_{0}\right\| \leq K\left\|h_{\delta} a_{0}-a_{0}\right\|\left\|x_{0}\right\| \rightarrow 0 .
$$

Now suppose that $\mathscr{f} x$ is closed in $X$. Let $a \in \mathfrak{A}$. Then $a x=\lim a h_{\delta} x$ and $a h_{\delta} x \in \mathscr{g}_{x}$ implies that $a x \in \overline{g_{x}}$. But $\overline{g_{x}}=g_{x}$ so $g_{x} \subset \mathfrak{U} x \subset g_{x}$ and equality follows.

Suppose now that $\mathfrak{A} x$ is closed in $X$. Let $\mathscr{G}=\{a \in \mathfrak{A}: a x=0\}$. Then the map $a+G \rightarrow a x$ is a one-to-one continuous linear map of $\mathfrak{A} / \mathcal{G}$ onto $\mathfrak{A} x$. By the open mapping theorem, there exists a constant $c>0$ such that $\|a+\mathscr{I}\| \leq c\|a x\|$ for all $a \in \mathfrak{A}$. Let $h \in\left\{h_{\delta}\right\}_{\delta \in \Lambda}$ be such that $\|h x-x\|<$ 1/4Kc. Consider the map $T: a \rightarrow a h x$. Then $T$ is a continuous map of $\mathfrak{A}$ into $\mathfrak{A} x$. We show that $T$ is surjective. Let $a x \in \mathfrak{A} x,\|a x\| \leq 1$. Then $\|a+9\| \leq c$ so there exists $b \in \mathfrak{A}$ such that $\|a+b\| \leq 2 c$. Then

$$
\begin{aligned}
\|T(a+b)-a x\| & =\|(a+b) h x-a x\|=\|(a+b) h x-(a+b) x\| \\
& \leq K\|a+b\|\|h x-x\|<K(2 c) / 4 K c=1 / 2 .
\end{aligned}
$$

So by Lemma 3.1, $T$ is subjective and therefore $\mathfrak{A} x=\mathscr{g} h x$. But $\mathfrak{U} h \subset \mathscr{g}$, therefore $\mathfrak{A} x=\mathfrak{A} h x \subset \mathscr{f} x \subset \mathfrak{A} x$ and equality holds.

We can identify $l_{1}(G)$ as a closed ideal of $M(G)$ by considering a function $f \in L_{1}(G)$ as the finite regular Borel measure $f(x) d x$. Furthermore, as is shown in Hewitt and Ross [7, 28.52], $L_{1}(G)$ has a bounded approximate identity. We can now use Lemma 3.2 to extend Theorem 1.1 to $M(G)$.

Corollary 3.1. (i) Let $f \in L_{p}(G), 1<p<\infty, f \neq 0$. Then $M(G) * f$ is not closed in $L_{p}(G)$.

(ii) Let $f \in C_{0}(G)$. Then $M(G) * f$ is not closed in $C_{0}(G)$.

Proof. It follows from the factorization theorem, Hewitt and Ross [7, 32.22], that $L_{1}(G) * L_{p}(G)=L_{p}(G)$ and $L_{1}(G) * C_{0}(G)=C_{0}(G)$. Also since $M(G) * L_{\mathrm{l}}(G)=L_{\mathrm{l}}(G)$ we get that

$$
M(G) * C_{0}(G)=M(G) * L_{1}(G) * C_{0}(G)=L_{1}(G) * C_{0}(G)=C_{0}(G) .
$$

We apply Lemma 3.2 with $\mathfrak{A}=M(G), g=L_{1}(G)$ and in (i) $X=L_{p}(G)$, $1<p<\infty$, and in (ii) $X=C_{0}(G)$ to conclude that if $M(G) * f$ is closed then so is $L_{1}(G) * f$. But this is impossible by Theorem 1.1.

EXAMPLE 2.1. When $p=1$ and $G=\mathbf{Z}$, we have $M(\mathbf{Z})=l_{1}(\mathbf{Z})$, so as in Example 1.1, $M(\mathbf{Z}) * \delta_{0}=l_{1}(\mathbf{Z})$, which is closed. 
4. Maps from $L_{p}(G)$ into $C_{0}(G)$. In this section we consider the closure of the range of operators from $L_{p}(G)$ to $C_{0}(G), 1<p<\infty$. These operators are of the form $T_{\tilde{f}}$, convolution on the right by $\tilde{f}$, where $f \in L_{p^{\prime}}(G)$, and $\tilde{f}(x)=f\left(x^{-1}\right), p^{\prime}=p /(1-p)$. It follows from (3) that $T_{\tilde{f}}$ is a bounded operator with domain $L_{p}(G)$ and range $C_{0}(G)$.

THEOREM 4.1. Let $1<p<\infty, p^{\prime}=p /(1-p)$ and $f \in L_{p^{\prime}}(G), f \neq 0$. Then $L_{p}(G) * \tilde{f}$ is not closed in $C_{0}(G)$.

Proof. The proof is a consequence of Corollary 3.1 (ii). The range of the map $T_{\tilde{f}}$ of $L_{p}(G)$ into $C_{0}(G)$ is $L_{p}(G) * \tilde{f}$. Suppose $T_{\tilde{f}}$ has closed range. Let $\mathcal{L}=T_{\tilde{f}}^{-1}\{0\}$ be a closed subspace of $L_{p}(G)$ so as in the proof of Theorem 1.1 the map $\Phi: L_{p}(G) / \mathcal{L} \rightarrow C_{0}(G)$ defined by $\Phi(g+\mathcal{L})=g * \tilde{f}$ is a continuous one-to-one map of the Banach space $L_{p}(G) / \mathcal{L}$ onto the Banach subspace $L_{p}(G) * f$ of $C_{0}(G)$. The open mapping theorem implies that $\Phi$ is bicontinuous. Again as in the proof of Theorem $1.1\left(L_{p}(G) / \varrho\right)^{*}$ is isometrically isomorphic with the subspace $\mathcal{L}^{\perp}$ of $L_{p^{\prime}}(G)$, where $\mathcal{L}^{\perp}=\{h$ $\in L_{p^{\prime}}(G):\langle g, h\rangle=0$ for all $\left.g \in \mathcal{E}\right\}$. So the adjoint of $\Phi, \Phi^{*}$, is a bicontinuous map from $\left[L_{p}(G) * \tilde{f}\right]^{*}$ onto $\mathcal{L}^{\perp}$. Now consider the injection map, $i$, of $L_{p}(G) * \tilde{f}$ into $C_{0}(G)$. Since $L_{p}(G) * \tilde{f}$ is closed, the adjoint of $i$, $i^{*}$, maps $M(G)=\left(C_{0}(G)\right)^{*}$ onto $\left[L_{p}(G) * \tilde{f}\right]^{*}$, see Hewitt and Ross [7, E.8]. It follows that the composition map $\Phi^{*} \circ i^{*}(\mu)$ is a continuous map of $M(G)$ onto the closed subspace of $L_{p^{\prime}}(G)$. We compute $\Phi^{*} \circ i^{*}(\mu)$ for a fixed $\mu \in M(G)$. Since $\Phi^{*} \circ i^{*}(\mu) \in L_{p^{\prime}}(G)$ and $L_{p^{\prime}}(G)=\left[L_{p}(G)\right]^{*}$, it's enough to evaluate $\Phi^{*} \circ i^{*}(\mu)$ acting as a functional on an arbitrary $g$ in $L_{p}(G)$.

$$
\begin{aligned}
\int g(x) \overline{\Phi^{*} \circ i^{*}(\mu)}(x) d x & =\left\langle(g+\mathcal{L}), i^{*}(\mu)\right\rangle \\
& =\left\langle g * \tilde{f}, i^{*}(\mu)\right\rangle=\langle g * \tilde{f}, \mu\rangle \\
& =\int g * \tilde{f}(x) d \bar{\mu}(x) \\
& =\iint g(y) \tilde{f}\left(y^{-1} x\right) d y d \bar{\mu}(x) \\
& =\iint g(y) f\left(x^{-1} y\right) d \bar{\mu}(x) d y \\
& =\int g(y)\left[\int f\left(x^{-1} y\right) d \bar{\mu}(x)\right] d y \\
& =\int g(y)(\mu * \bar{f})^{-}(y) d y .
\end{aligned}
$$


Therefore $\Phi^{*} \circ i^{*}(\mu)=\mu * \bar{f}$ a.e. However $\mathcal{L}^{\perp}$ is the range of $\Phi^{*} \circ i^{*}$ so the above computation shows that $\mathfrak{L}^{\perp}=M(G) * \bar{f}$ and this subspace is closed in $L_{p^{\prime}}(G)$. By Corollary 3.1 (i) this is impossible. Therefore the original assumption, that $L_{p}(G) * \tilde{f}$ is closed in $C_{0}(G)$ can't hold. This proves the theorem.

EXAMPLE 4.1. Formula (4) shows that if $f \in L_{\infty}(G)$ then the operator $T_{f}$ is a bounded linear operator from $L_{1}(G)$ to $C(G)$. If we let $f$ be identically 1 , then for $g \in L_{1}(G), T_{\tilde{f}}(g)(x)=g * 1(x)=\int g(y) d y$. Therefore the range of $T_{1}, L_{1}(G) * 1$, is $\{\alpha 1: \alpha \in \mathbf{C}\}$ which is closed in $C(G)$.

Formula (5) shows that if $f \in L_{1}(G)$ then the operator $T_{\tilde{f}}$ is a bounded linear operator from $L_{\infty}(G)$ to $C(G)$. Let $G=\mathbf{Z}$ and $f=\delta_{0}$, point mass at 0 . Then $\tilde{\delta_{0}}=\delta_{0}$ and $l_{\infty}(\mathbf{Z})=c(\mathbf{Z})$ so $l_{\infty}(\mathbf{Z}) * \delta_{0}=c(\mathbf{Z})$ is closed.

5. Maps from $L_{p}(G)$ into $L_{r}(G)$. The last convolution formula is (6). From it we see that if $p, q>1,1 / p+1 / q-1 / r=1$ and $f \in L_{q}(g)$ $\cap\left[L_{q}(G)\right]^{\tilde{y}}$, then $T_{f}$ is a bounded operator from $L_{p}(G)$ into $L_{r}(G)$. We are interested in whether these operators can have closed range. It will turn out that if $f \neq 0$, the range of $T_{f}$ is not closed. Before proving this we need a lemma which is similar in idea to Lemma 1.1, but the details of the proof are somewhat different.

First note that since $p, q>1$, we have $2-1 / r>1 / p+1 / q-1 / r$ $=1$ and so $r>1$. Let $p^{\prime}, q^{\prime}$ and $r^{\prime}$ be the conjugate exponents of $p, q$ and $r$ respectively. Let $f \in L_{q}(G) \cap\left[L_{q}(G)\right]^{\tilde{y}}, f \neq 0$, and let $M=L_{p}(G) * f$. Then (6) implies that $M \subset L_{r}(G)$. Also

$$
1 / p+1 / p^{\prime}+1 / q+1 / q^{\prime}-1 / r-1 / r^{\prime}=-1 / r-1 / r^{\prime}=1
$$

and

$$
1 / p+1 / q-1 / r=1
$$

imply that

$$
1 / p^{\prime}+1 / g^{\prime}-1 / r^{\prime}=0
$$

So

$$
1 / r+1 / g-1 / p^{\prime}=1 / q+\left(1 / r^{\prime}-1 / p^{\prime}\right)=1 / q+1 / q^{\prime}=1 .
$$

Therefore by applying (6) we get $\varphi * \tilde{\tilde{f}} \in L_{p}^{\prime}(G)$ for all $\varphi \in L_{r}^{\prime}(G)$ and

$$
\|\varphi * \tilde{\tilde{f}}\|_{p^{\prime}} \leq\|\varphi\|_{r^{\prime}}\|\tilde{\tilde{f}}\| q .
$$


LEMMA 5.1. Let $p, q$ and $r$ be as above, $f \in L_{q}(G) \cap\left[L_{q}(G)\right]^{\tilde{}}, f \neq 0$, and $M=L_{p}(\underline{\tilde{g}}) * f$. Then there exists $\left\{\varphi_{n}\right\}_{n=1}^{\infty} \subset L_{r}^{\prime}(G)$ such that $\left\|\varphi_{n}\right\|_{M^{*}} \rightarrow$ $\infty$ and $\left\|\varphi_{n} * \tilde{f}\right\|_{p^{\prime}}$ is bounded.

Proof. Fix $n$. We saw that $M \subset L_{r}(G)$, so pick $k \in M,\|k\|_{r}=1$. By the Hahn-Banach Theorem, there exists $h \in L_{r^{\prime}}(G),\|h\|_{r^{\prime}}=1$ such that $\langle k, \bar{h}\rangle=1$. The remarks before the statement of the lemma show that $h * \tilde{f} \in L_{p^{\prime}}(G)$.

Choose $k_{0}$ and $g_{0}$ in $C_{00}(G)$ such that

$$
\left\|k-k_{0}\right\|_{r}<1 / n \text { and }\left\|h * \tilde{\tilde{f}}-g_{0}\right\|_{p^{\prime}}<\|\tilde{\tilde{f}}\|_{q} / n \text {. }
$$

By (3) $h * \tilde{k} \in C_{0}(G)$. Just as we did in the proof of Lemma 1.1, we can pick $x_{i}, \ldots, x_{n}$ in $G$ such that:

(i) the supports of the functions $x_{i} k_{0}, i=1, \ldots, n$, are disjoint;

(ii) $\left|\bar{h} * \tilde{f}\left(x_{i} x_{j}^{-1}\right)\right|<1 / n(n-1)$ for $i \neq j$; and

(iii) the supports of the functions $x_{i} g_{0}, i=1, \ldots, n$, are disjoint.

Since $1 / p+1-1 / r>1 / p+1 / q-1 / r=1$ we get that $r>p$ and so $r^{\prime}<p^{\prime}$. Let $s$ be any number such that $r^{\prime}<s<p^{\prime}$. And let $\varphi_{n}=$ $\sum_{j=1}^{n} j^{-1 / s} x_{j} h$. Then $\varphi_{n} \in M$ and

$$
\begin{aligned}
& \left|\left\langle\sum_{i=1}^{n} x_{i} k, \varphi_{n}\right\rangle\right|=\left|\int \varphi_{n}(x) \sum_{i=1}^{n} x_{i} k(x) d x\right| \\
& \quad=\left|\sum_{j=1}^{n} j^{-1 / s} \int x_{j} \bar{h}(x) x_{j} k(x) d x+\sum_{i \neq j}(i j)^{-1 / s} \int x_{i} \bar{h}(x) x_{j} k(x) d x\right| \\
& =\left|\sum_{j=1}^{n} j^{-1 / s}+\sum_{i \neq j}(i j)^{-1 / s} \bar{h} * \tilde{k}\left(x_{i} x_{j}^{-1}\right)\right| \\
& \geq \sum_{j=1}^{n} j^{-1 / s}-\sum_{i \neq j}\left|\bar{h} * \tilde{k}\left(x_{i} x_{j}^{-1}\right)\right| \\
& \geq \sum_{j=1}^{n} j^{-1 / s}-1=\sum_{j=2}^{n} j^{-1 / 2} .
\end{aligned}
$$

Since the supports of the $x_{i} k_{0}$ are disjoint, and $\left\|k_{0}\right\|_{r} \leq 2$ we get

$$
\left\|\sum_{i=1}^{n} x_{i} k_{0}\right\|_{r}^{r} \leq 2^{r} n .
$$


Therefore as in the proof of Lemma 1.1,

$$
\begin{aligned}
\left\|\sum_{i=1}^{n} x_{i} k\right\|_{r} & \leq\left\|\sum_{i=1}^{n} x_{i} k-\sum_{i=1}^{n} x_{i} k_{0}\right\|_{r}+\left\|\sum_{i=1}^{n} x_{i} k_{0}\right\|_{r} \\
& \leq 1+2 n^{1 / r} \leq 3 n^{1 / r} .
\end{aligned}
$$

We have $\sum_{i=1}^{n} x_{i} k \in M$ since $M$ is closed under left translation. So a lower bound for $\left\|\varphi_{n}\right\|_{M^{*}}$ can be computed by

$$
\left\|\varphi_{n}\right\|_{M^{*}} \geq\left|\left\langle\frac{1}{3 n^{1 / r}} \sum_{i=1}^{n} x_{i} k, \varphi_{n}\right\rangle\right| \geq \frac{1}{3 n^{1 / r}} \sum_{j=2}^{n} j^{-1 / s}=\tau(n) .
$$

The fact that $\tau(n) \rightarrow \infty$ follows from the integral inequality.

$$
\frac{1}{z^{1 / r}} \int_{1}^{z} t^{-1 / s} d t \geq z^{1-(1 / r+1 / s)}-1
$$

and the fact that $s>r^{\prime}$ implies $1 / s+1 / r<1$.

Now in $L_{p}^{\prime}(G)$,

$$
\begin{aligned}
\|g\|_{p^{\prime}} & \leq\|h * \tilde{\tilde{f}}-g\|_{p^{\prime}}+\|h * \tilde{\tilde{f}}\|_{p^{\prime}} \\
& <\|f\|_{q} / n+\|h\|_{r^{\prime}}\|\tilde{\tilde{f}}\|_{q}<2\|\tilde{\tilde{f}}\|_{q},
\end{aligned}
$$

and

$$
\begin{aligned}
\left\|\varphi_{n} * \tilde{\tilde{f}}\right\|_{p^{\prime}} & =\left\|\left[\sum_{j=1}^{n} j^{-1 / s} x_{j} h\right] * \tilde{\tilde{f}}\right\|_{p^{\prime}}=\left\|\sum_{j=1}^{n} j^{-1 / s} x_{j}(h * \tilde{\tilde{f}})\right\|_{p^{\prime}} \\
& \leq\left\|\sum_{j=1}^{n} j^{-1 / s} x_{j}(h * \tilde{\tilde{f}})-\sum_{j=1}^{n} j^{-1 / s} x_{j} g\right\|_{p^{\prime}}+\left\|\sum_{j=1}^{n} j^{-1 / s} x_{j} g\right\|_{p^{\prime}} \\
& \leq \sum_{j=1}^{n} j^{-1 / s}\left\|x_{j}(h * \tilde{\tilde{f}})-x_{j} g\right\|_{p^{\prime}}+\left\|\sum_{j=1}^{n} j^{-1 / s} x_{j} g\right\|_{p^{\prime}} \\
& \leq 1+\left\|\sum_{j=1}^{n} j^{-1 / s} x_{j} g\right\|_{p^{\prime}}
\end{aligned}
$$

Since the supports of the $x_{j} g$ are disjoint,

$$
\left\|\sum_{j=1}^{n} j^{-1 / s} x_{j} g\right\|_{p^{\prime}}^{p^{\prime}}=\sum_{j=1}^{n} j^{-p^{\prime} / s}\left\|x_{j} g\right\|_{p^{\prime}}^{p^{\prime}}<2^{p^{\prime}}\|\tilde{\tilde{f}}\|_{q^{\prime}}^{p^{\prime}} \sum_{j=1}^{n} j^{-p^{\prime} / s} .
$$


Also since $p^{\prime} / s>1$ the series converges. Denote its limit by $\alpha$. So we get that

$$
\left\|\varphi_{n} * \tilde{\tilde{f}}\right\|_{p^{\prime}} \leq 1+2\|\tilde{\tilde{f}}\|_{q} \alpha^{1 / p^{\prime}} .
$$

This bound is independent at $n$. Therefore keeping $k$ and $h$ fixed for all $n$, and choosing appropriate $x_{l}, \ldots, x_{n}$ we can construct $\varphi_{n}$ with the desired properties. This proves the lemma.

THEOREM 5.1. Let $p, q>1,1 / p+1 / q-1 / r=1$ and $f \in L_{q}(G) \cap$ $\left[L_{q}(G) \tilde{]}, f \neq 0\right.$. Then $L_{p}(G) * f$ is not closed in $L_{r}(G)$.

Proof. The proof is identical to that of Theorem 1.1 (i) except here

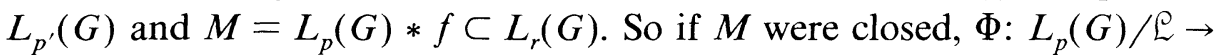
$M$ would be bicontinuous by the open mapping theorem and then so would $\Phi^{*}: M^{*} \rightarrow \mathfrak{L}^{\perp} \subset L_{p^{\prime}}(G)$. A similar computation to that in the proof of Theorem 1.1 (i) shows that when $h \in L_{r^{\prime}}(G), \Phi^{*}(h)=h * \tilde{\tilde{f}}$. But then Lemma 5.1 shows that there exists $\left\{\varphi_{n}\right\}_{n=1}^{\infty} \subset L_{r^{\prime}}(G)$ such that $\left\|\varphi_{n}\right\|_{M^{*}} \rightarrow \infty$ but $\left\|\Phi^{*}\left(\varphi_{n}\right)\right\|_{p^{\prime}}$ is bounded. This is impossible if $\Phi^{*}$ is bicontinuous. Therefore $M$ can't be closed.

For an alternate way of stating Lemma 5.1, Theorem 5.1 and formula (6) the reader is referred to the paper by B. Russo and A. Klein [8].

\section{REFERENCES}

[1] W. Bade and P. C. Curtis, Embedding theorems for commutative banach algebra, Pacific J. Math., 18 (1966), 391-409.

[2] R. Bekes, Algebraically irreducible representations of $L_{1}(G)$, Pacific J. Math., 60 (1975), 11-25.

[3] N. Dunford and J. T. Schwartz, Linear Operators, Part I, Interscience, New York 1958.

[4] I. Glicksberg, When is $L_{1}$ closed, Trans. Amer. Math. Soc., 160 (1971), 419-425.

[5] F. Greenleaf and M. Moskowitz, Cyclic vectors for representations associated with positive definite measures: Nonseparable groups, Pacific J. Math., 45 (1973), 165-186.

[6] E. Hewitt and K. A. Ross, Abstract Harmonic Analysis I, Springer-Verlag, Berlin, 1963.

[7] __ Abstract Harmonic Analysis II, Springer-Verlag, Berlin, 1970.

[8] B. Russo and A. Klein, Sharp Inequalities for Weyl Operators, Math. Ann., 235 (1978), 175-194.

Received June 18, 1981 and in revised form May 25, 1982.

The Colorado College

Colorado Springs, CO 80903 



\title{
PACIFIC JOURNAL OF MATHEMATICS EDITORS
}

\author{
DONALD BABBITT (Managing Editor) \\ University of California \\ Los Angeles, CA 90024 \\ Hugo Rossi \\ University of Utah \\ Salt Lake City, UT 84112 \\ C. C. MOORE and Arthur Ogus \\ University of California \\ Berkeley, CA 94720
}

\author{
J. Dugundir \\ Department of Mathematics \\ University of Southern California \\ Los Angeles, CA 90089-1113
}

R. FINN and H. SAMELSON

Stanford University

Stanford, CA 94305

ASSOCIATE EDITORS
R. ARENS
E. F. BECKENBACH
B. H. NeumanN
F. WOLF
K. YosHIDA (1906-1982)

\section{SUPPORTING INSTITUTIONS}

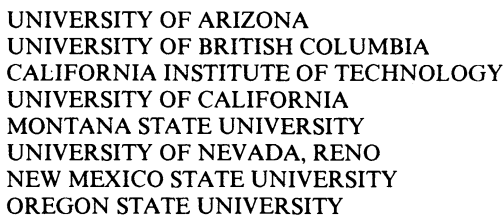

\author{
UNIVERSITY OF OREGON \\ UNIVERSITY OF SOUTHERN CALIFORNIA \\ STANFORD UNIVERSITY \\ UNIVERSITY OF HAWAII \\ UNIVERSITY OF TOKYO \\ UNIVERSITY OF UTAH \\ WASHINGTON STATE UNIVERSITY \\ UNIVERSITY OF WASHINGTON
}

The Supporting Institutions listed above contribute to the cost of publication of this Journal, but they are not owners or publishers and have no responsibility for its content or policies.

Mathematical papers intended for publication in the Pacific Journal of Mathematics should be in typed form or offset-reproduced (not dittoed), double spaced with large margins. Please do not use built up fractions in the text of the manuscript. However, you may use them in the displayed equations. Underline Greek letters in red, German in green, and script in blue. The first paragraph must be capable of being used separately as a synopsis of the entire paper. In particular it should contain no bibliographic references. Please propose a heading for the odd numbered pages of less than 35 characters. Manuscripts, in triplicate, may be sent to any one of the editors. Please classify according to the scheme of Math. Reviews, Index to Vol. 39. Supply name and address of author to whom proofs should be sent. All other communications should be addressed to the managing editor, or Elaine Barth, University of California, Los Angeles, California 90024.

There are page-charges associated with articles appearing in the Pacific Journal of Mathematics. These charges are expected to be paid by the author's University, Government Agency or Company. If the author or authors do not have access to such Institutional support these charges are waived. Single authors will receive 50 free reprints; joint authors will receive a total of 100 free reprints. Additional copies may be obtained at cost in multiples of 50 .

The Pacific Journal of Mathematics is issued monthly as of January 1966. Regular subscription rate: \$132.00 a year (6 Vol., 12 issues). Special rate: $\$ 66.00$ a year to individual members of supporting institutions.

Subscriptions, orders for numbers issued in the last three calendar years, and changes of address should be sent to Pacific Journal of Mathematics, P.O. Box 969, Carmel Valley, CA 93924, U.S.A. Old back numbers obtainable from Kraus Periodicals Co., Route 100, Millwood, NY 10546.

The Pacific Journal of Mathematics ISSN 0030-8730 is published monthly by the Pacific Journal of Mathematics at P.O. Box 969, Carmel Valley, CA 93924. Application to mail at Second-class postage rates is pending at Carmel Valley, California, and additional mailing offices. Postmaster: Send address changes to Pacific Journal of Mathematics, P. O. Box 969, Carmel Valley, CA 93924.

PUBLISHED BY PACIFIC JOURNAL OF MATHEMATICS, A NON-PROFIT CORPORATION

Copyright $(1984$ by Pacific Journal of Mathematics 


\section{Pacific Journal of Mathematics}

Vol. 110, No. $2 \quad$ October, 1984

Robert A. Bekes, The range of convolution operators $\ldots \ldots \ldots \ldots \ldots 257$

Dennis K. Burke and Sheldon Davis, Subsets of ${ }^{\omega} \omega$ and generalized metric

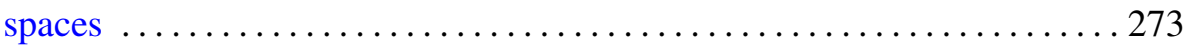

Giovanni Emmanuele, A remark on a paper: "Common fixed points of nonexpansive mappings by iteration" $\ldots \ldots \ldots \ldots \ldots \ldots \ldots \ldots \ldots 283$

I. Erdélyi and Sheng-Wang Wang, On strongly decomposable operators . . . 287

Gerhard Gierz, Injective Banach lattices with strong order units . . . . . . . 297

Maurizio Letizia, Quotients by complex conjugation of nonsingular quadrics and cubics in $\mathbf{P}_{\mathbf{C}}^{3}$ defined over $\mathbf{R} \ldots \ldots \ldots \ldots \ldots \ldots \ldots \ldots \ldots \ldots$

P. H. Maserick and Franciszek Hugon Szafraniec, Equivalent definitions

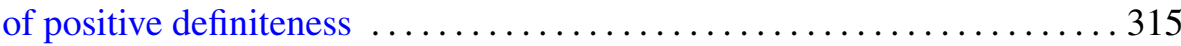

Costel Peligrad and S. Rubinstein, Maximal subalgebras of $C^{*}$-crossed

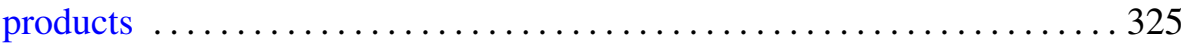

Derek W. Robinson and Sadayuki Yamamuro, Hereditary cones, order

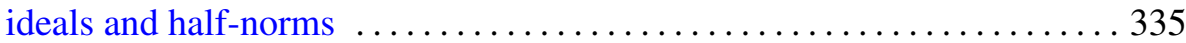

Derek W. Robinson and Sadayuki Yamamuro, The Jordan decomposition

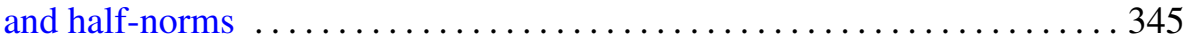

Richard Rochberg, Interpolation of Banach spaces and negatively curved

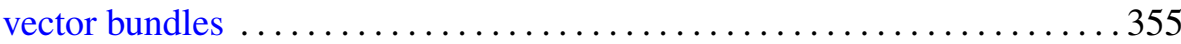

Dale Rolfsen, Rational surgery calculus: extension of Kirby's theorem 377

Walter Iaan Seaman, Helicoids of constant mean curvature and their Gauss maps

Diana Shelstad, Endoscopic groups and base change $\mathbf{C} / \mathbf{R}$

Jerrold Norman Siegel and Frank Williams, Numerical invariants of homotopies into spheres

Alladi Sitaram, Some remarks on measures on noncompact semisimple Lie groups

Teruhiko Soma, Atoroidal, irreducible 3-manifolds and 3-fold branched coverings of $S^{3}$

Jan de Vries, On the $G$-compactification of products

Hans Weber, Topological Boolean rings. Decomposition of finitely additive set functions 